\title{
The twists and turns of chiral chemistry
}

\author{
Chirality is fundamental to chemistry, molecular biology and photonics, but chirality itself is not often in the \\ spotlight. Lewis E. MacKenzie and Patrycja Stachelek report on the 2021 Chiral Materials meeting, which explored \\ how chirality manifests in functional materials, and how it can lead to new technological applications.
}

$\mathrm{C}$ hirality - where an object cannot be superimposed on its mirror image - manifests across all size scales: from fundamental particles to galactic structures. Perhaps most prominently, chirality underpins chemical interactions: twisted molecular structures will interlock and bind only if their chirality allows them. This in turn makes chirality fundamental to the processes of life. Chirality also drives supramolecular and bulk material properties, with chiral molecular arrangements altering material conductivity or the handedness of emitted circularly polarized photons. Chirality manipulation therefore gives new ways to tune and optimize material properties for organic electronics and 3D display technology applications. Yet, whilst chiral phenomena are at the heart of a vast array of sciences, most notably chemistry and biology, chirality itself is not often the focus of attention.

'Chiral Materials' is a new and independent series of researcher-led meetings that put chirality centre stage. It aspires to link the community of researchers across the globe who study chirality, albeit with the majority of speakers hailing from the UK and Europe, to explore the varied phenomena and applications that emerge from the topic. This year's meeting was the second such event (the first was held in 2019) and was co-organized by Jessica Wade (Imperial College London), Paul McGonigal and Robert Pal (both Durham University). Due to the COVID-19 crisis, this Chiral Materials meeting was held via Zoom on 23 March 2021 after being delayed from 2020.

Although many will be disappointed to not have had the opportunity to meet their colleagues in person, the online nature of the event made it easy for over 370 registered attendees from around the world to attend live talks as well as a poster session. Guest speakers discussed their recent research into the emergent properties of small molecules, thin films, polymers, structural chirality, chiral matter and light interactions, as well as new technological developments in spectroscopy and computational screening techniques (Fig. 1).
The first session of the day focused on the synthesis and design of chiral molecules. Helicenes - finding use in a variety of applications - were a recurring theme. For example, Matthew Fuchter (Imperial College London) described recent advances in combining helicenes with achiral luminescent polymers, to generate unprecedented strong circularly polarized luminescence (CPL) emission via natural optical activity (rather than structural chirality as previously assumed). This approach is particularly promising for the development of a new-generation organic light emitting diodes (OLEDs) ${ }^{1}$. Michal Juríček (University of Zurich) presented how organic chiral molecules (such as helicenes and helical cethrenes) can be utilized as magnetic switches and chiroptical photoswitches with impressive experimental demonstrations ${ }^{2}$. Steve Goldup (University of Southampton) described advances in processes for the stereoselective synthesis of mechanically chiral systems (for example, mechanically interlocked molecules) that can be used as enantioselective catalysis ${ }^{3}$. The session closed with Alyssa-Jennifer Avestro (University of York), describing recent research into the structure and properties of helicenes.

The lunch break afforded the opportunity to attend the virtual poster session. Many of the posters included a short ( $<1$ minute) video introduction by the presenter and the posters themselves were in PDF format. The video introductions made the work very accessible and this innovation would no doubt be welcome at future conferences, whether virtual or in person. Poster prizes were awarded by attendee voting. Carin Lightner (ETH Zurich), Leah White (University of Bath), Lizzie Killalea (University of Nottingham) and Marco Preuss (Eindhoven University of Technology) all won prizes.

The early afternoon session focused on chiral characterization techniques. Kim Jelfs (Imperial College London) outlined how computational approaches can be used to rapidly predict/screen the properties of bulk materials assembled from chiral constituents. This impressive approach enables prediction of material properties (for example, to optimize conductivity) and has been experimentally validated ${ }^{4}$. Both Lorenzo Di Bari and Francesco Zinna (University of Pisa) discussed recent work on the structure and chiroptical properties arising from chiral organic thin films ${ }^{5,6}$. The session closed with Lewis MacKenzie (Durham University) describing the development a new rapid CPL spectrometer for situations where rapid analysis is required, for example, verification of CPL-active luminescent security inks ${ }^{7}$.

The final session centred on applications, with a heavy slant towards the nanoscale. Ventsislav K. Valev (University of Bath) described hyper-Rayleigh scattering interactions between nanoscale helicoids and circularly polarized light ${ }^{8}$. Malcolm Kadodwala (University of Glasgow) described how spin angular momentum from circularly polarized light can be transferred to nanofilms, resulting in remarkable reversible control of symmetry in solids ${ }^{9}$. Bart Kahr (New York University) described work in unpicking scale-dependent crystal growth mechanisms, which - much to the interest of the audience - are surprisingly chiral at small scales ${ }^{10}$.

The meeting was rounded off by A. P. de Silva (Queen's University Belfast), whose talk focused on the 'big picture' stories of his career as a scientist. Amongst many topics, he touched on the science of photoinduced electron-transfer sensors and functional image processing with chemicals ${ }^{11}$. Importantly, de Silva emphasized how kindness, mentorship and serendipity are hugely important in scientific research.

At the end of the day, it felt odd to simply switch off from the virtual meeting with no informal farewells or after-conference dinner. However, the global accessibility of this free meeting was certainly an advantage, and virtual posters with accompanying video introductions can still be found on the 2021 Chiral Materials website (https://www. chiralmaterials.co.uk/). We look forward to more Chiral Materials meetings in future years, whether they are virtual, in person, or a combination of the best aspects of both meeting formats. 
a

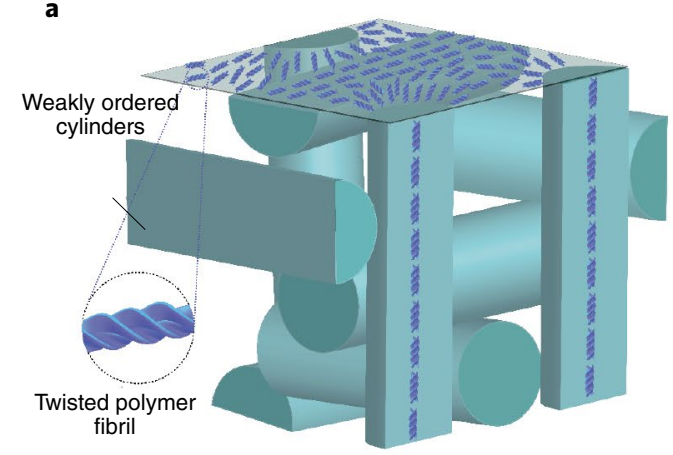

b

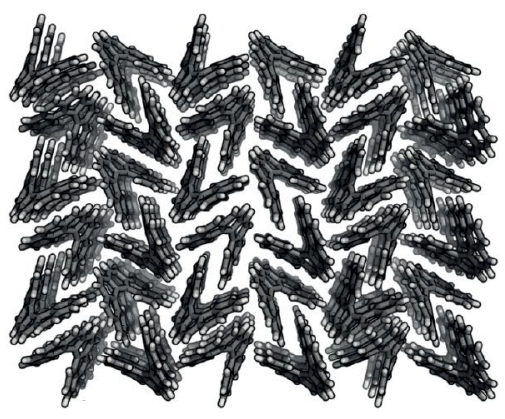

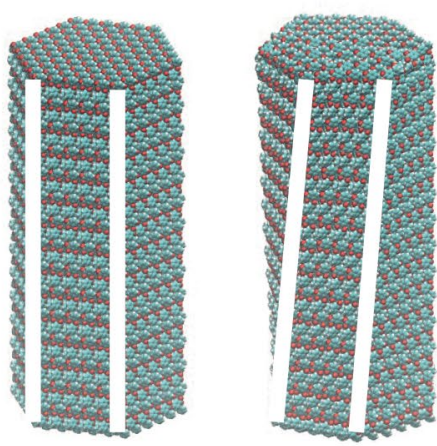
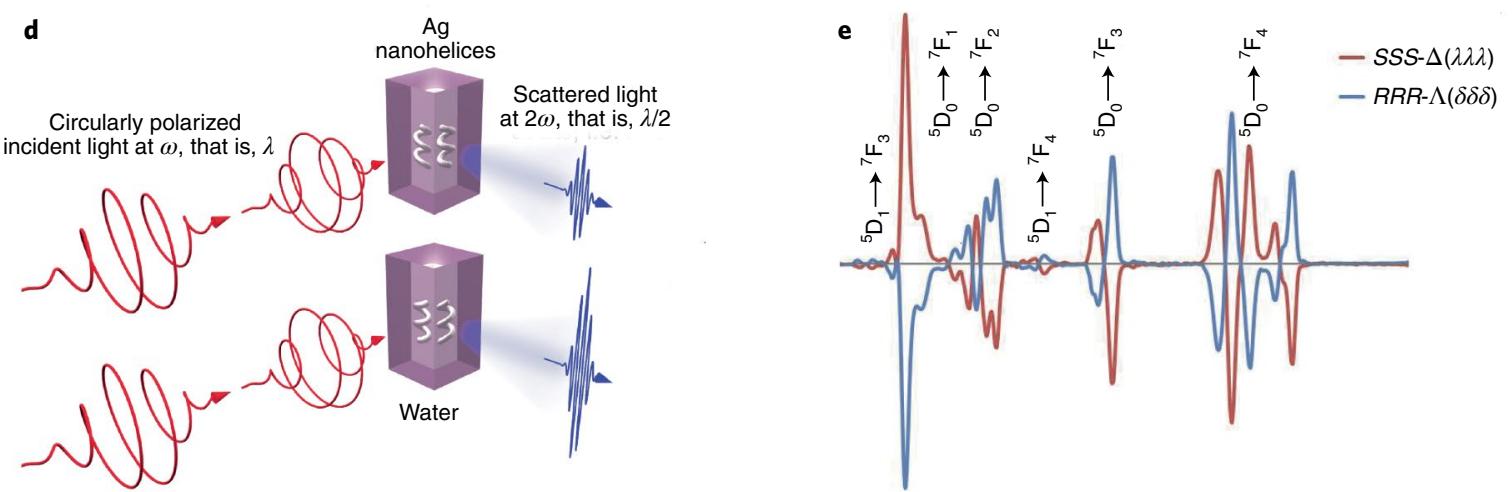

Fig: 1 | Some of the chiral materials and chiral phenomena presented at Chiral Materials 2021. a, Recent investigations have shown that the large chiroptical effects that manifest in polymer thin films formed from achiral polymers with chiral additives (that is, 1-aza[6] helicene) arise due to magneto-electric coupling (natural optical activity), not structure chirality as previously assumed'. Image provided by Jessica Wade and Matthew Fuchter. b, Varying chiral molecular packing in organic semiconductors alters the charge-carrier mobility properties of the material; computational approaches can be used for mass screening of many configurations to optimize properties such as charge mobility ${ }^{4}$. Image provided by Kim Jelfs. c, Chiral benzyl nanorods arise through spontaneous nanoscale twisting mechanisms ${ }^{10}$. Image provided by Bart Kahr. $\mathbf{d}$, A new chiroptical effect: nonlinear optical hyper-Rayleigh scattering at the second harmonic light frequency is dependent on the chirality of $\mathrm{Ag}$ nanohelices dispersed in an isotropic liquid ${ }^{8}$. Image provided by Ventsislav Valev. e, Circular polarized luminescence spectroscopy enables elucidation of excited-state properties of luminescent chiral molecules for applications such as advanced security inks? Image provided by Robert Pal.

Lewis E. MacKenzie ${ }^{\bowtie}$ and Patrycja Stachelek Department of Chemistry, Durham University, Durham, UK.

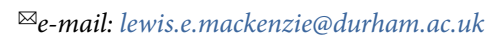

Published online: 1 June 2021

https://doi.org/10.1038/s41557-021-00729-8
References

1. Wade, J. et al. Nat. Commun. 11, 6137 (2020).

2. Ravat, P., Šolomek, T. \& Juríček, M. ChemPhotoChem. 3, 180-186 (2019).

3. Maynard, J. R. J. \& Goldup, S. M. Chem. 6, 1914-1932 (2020).

4. Schmidt, J. A. et al. Preprint at ChemRxiv https://doi. org/10.26434/chemrxiv.12451943 (2021).

5. Albano, G., Pescitelli, G. \& Di Bari, L. Chem. Rev. 120, 10145-10243 (2020)

6. Zinna, F. et al. Adv. Mater. 32, 2002575 (2020).
7. MacKenzie, L., Pålsson, L.-O., Parker, D., Beeby, A. \& Pal, R. Nat Commun 11, 1676 (2020).

8. Collins, J. T. et al. Phys. Rev. X 9, 011024 (2019).

9. Kelly, C. et al. Nat. Commun. 11, 5169 (2020).

10. Li, C. et al. J. Phys. Chem. C 124, 15616-15624 (2020).

11. Ling, J., Naren, G., Kelly, J., Moody, T. S. \& de Silva, A. P. J. Am. Chem. Soc. 137, 3763-3766 (2015).

Competing interests

The authors declare no competing interests. 